\title{
El análisis del disfrute y la ansiedad como fundamento metodológico en las clases de adultos
}

\section{Irene Acosta-Manzano}

Profesora de la Escuela Oficial de Idiomas de Lucena y estudiante de Doctorado de la Universidad de Málaga (ireneacostamanzano@gmail.com)

\begin{abstract}
Research on the field of positive and negative emotions in Adult Foreign Language Learning is still scarce. For this reason, a Working Group at the State Language School of Lucena launched a project to further their knowledge of the Foreign Language Enjoyment and Foreign Language Classroom Anxiety that adult students feel during the foreign language learning process. By analyzing adult students' emotions, teachers have the tools to substantiate their methodological decisions and improve the education system. In this study, only the results of the 25 B2.2. English learners are examined out of the 190 participants who filled the questionnaire. To promote participation, the questionnaire was distributed through Google Forms and published on the teachers' online learning platforms. Time was also assigned to fill it in class. The data analysis was carried out with the software IBM SPSS Statistics. Thanks to this study, the different conditions that trigger positive and negative emotions in adult students have been identified and methodologies can be adaptded to improve the adult foreign language teaching-learning process.
\end{abstract}

Keywords: Foreign Language Enjoyment, Foreign Language Classroom Anxiety, methodology, adult students, B2.2., English as a Foreign Language.

\section{Resumen}

El campo de las emociones tanto positivas como negativas en el aprendizaje de lengua extranjera por adultos se caracteriza por la escasez de investigaciones. Por este motivo, se inició un Grupo de Trabajo en la Escuela Oficial de Idiomas de Lucena que indagó en el disfrute y la ansiedad que el alumnado adulto experimenta durante el proceso de enseñanza-aprendizaje de una lengua extranjera con el fin de fundamentar las decisiones metodológicas del profesorado y contribuir a la mejora de la calidad educativa. En este estudio se analizan concretamente los resultados de los 25 aprendientes de Nivel Intermedio B2.2. de inglés de los 190 participantes que rellenaron el cuestionario. Para fomentar la participación, el cuestionario se distribuyó a través de un enlace de Formularios de Google, se publicó en las plataformas de aprendizaje virtuales y se dejó tiempo en clase para cumplimentarlo. El análisis de los datos se llevó a cabo mediante el programa IBM SPSS Statistics. Gracias a este estudio, se pudieron identificar las fuentes que ocasionan FLE y FLCA y adaptar la metodología para mejorar el proceso de enseñanza-aprendizaje de lenguas extranjeras en adultos.

Palabras clave: disfrute, ansiedad, metodología, alumnado adulto, B2.2., Inglés como Lengua Extranjera 


\section{Introducción}

La investigación acerca de las perspectivas sobre el disfrute y la ansiedad de estudiantes adultos de Lengua Extranjera (LE) es aún escasa en nuestro entorno. Este estudio contribuye a paliar esta situación y permite identificar algunas de las fuentes que los ocasionan en el aula con el objetivo de fundamentar las decisiones metodológicas del profesorado, fomentar las experiencias positivas y disminuir las negativas.

La ansiedad en la clase de lengua extranjera (FLCA) se puede definir como la preocupación o la reacción emocional negativa causada durante la utilización de una lengua extranjera (MacIntyre, 1999). La FLCA se ha relacionado en la literatura con variables tales como la edad, el uso auténtico de la lengua, la frecuencia de uso, el nivel de socialización en la lengua extranjera (Dewaele, MacIntyre y Boudreau, 2016), el nivel de formación, el número de idiomas aprendidos anteriormente, la distancia tipológica entre la lengua nativa y la lengua meta (Dewaele y MacIntyre, 2016). Por ello, se incluyeron estos ítemes entre otros en el cuestionario que se distribuyó al alumnado.

El disfrute (FLE), por su parte, tiende a ir ligado al estudio de FLCA. La investigación de las emociones se solía focalizar en las emociones negativas. No obstante, en los últimos años, se ha empezado a indagar en las emociones positivas ( $\mathrm{Li}, 2019)$. Los autores, que más han profundizado en el campo del disfrute y la ansiedad en adolescentes y niños, como MacIntyre y Gregersen (2012, 2014), Dewaele y MacIntyre $(2016,2019)$ señalan la independencia y el dinamismo de estas dos nociones. En cuanto al disfrute más concretamente, enfatizan la importancia del contexto de aprendizaje y las variables por las que se ve afectado.

El disfrute y la ansiedad están presentes en todos los aprendientes de LE y se relacionan negativamente (Dewaele \& MacIntyre, 2016). La mayoría de los estudios relativos al papel de FLE y FLCA en la clase de lengua extranjera se han centrado en tres tendencias ( $\mathrm{Li}, 2019)$. La primera mide el nivel de FLE experimentado por el estudiantado a través de un cuestionario cuantitativo de 21 ítems. La segunda investiga las relaciones entre FLE, FLCA y una amplia gama de variables del aprendiente y del docente. La tercera examina los vínculos entre FLE, FLCA y rendimiento académico en la LE. Los resultados indican consistentemente que hay una relación negativa significativa entre el disfrute y la ansiedad (Dewaele and MacIntyre 2014, 2016; Dewaele and Dewaele 2017; Boudreau, MacIntyre, and Dewaele 2018; Dewaele and Alfawzan 2018; Saito et al. 2018). El instrumento de esta investigación se basa en las dos primeras tendencias, es decir, mide el nivel de FLE y FLCA e investiga su relación con diversas variables.

Entre los objetivos de este estudio destaca el fomento de las emociones positivas dado que dichas emociones positivas, como el disfrute (FLE), enriquecen las percepciones del alumnado, incrementan su implicación con la LE y les permiten la exploración de contextos desconocidos (Gregersen, MacIntyre, \& Meza, 2014). Asimismo, fomentan la construcción de recursos que pueden resultar útiles en el futuro, la ampliación de los repertorios de pensamiento-acción, y la enmienda de las consecuencias perjudiciales de las emociones negativas (Fredrickson, 2003). Según Pavelescu y Petric (2018), las emociones positivas fuertes y duraderas son cruciales puesto que contribuyen a la ampliación del conocimiento y a la implicación del alumnado incluso cuando tiene que realizar tareas más tediosas. Esta capacidad no se observa en el caso de aprendientes con emociones positivas menos fuertes (Pavelescu y Petric, 2018).

En la literatura sobre FLE y FLCA, se observa la focalización de la mayoría de los estudios en los rangos de edad más bajos, es decir, niños y adolescentes (Li, 2019; MacIntyre y Gregersen 2012, 2014; Dewaele y MacIntyre 2016, 2019). Por consiguiente, la escasa representatividad en los estudios de discentes de otros grupos de edad es destacable, particularmente la de adultos de edades superiores a las que 
típicamente tienen estudiantes universitarios. De ahí, la necesidad de explorar estas emociones en el alumnado de las Escuelas Oficiales de Idiomas.

Los resultados del análisis del disfrute y la ansiedad del alumnado adulto de la EOI de Lucena le permitió al profesorado desarrollar una metodología fundamentada que trata de incentivar el disfrute y disminuir la ansiedad. Asimismo, les permitió un conocimiento más profundo de su alumnado.

En conclusión, esta comunicación pretende contribuir a la ampliación del conocimiento en torno a la importancia del análisis del disfrute y la ansiedad experimentadas por una variedad más amplia de alumnado adulto durante el proceso de enseñanza aprendizaje de idiomas. Con dicho análisis, será posible fundamentar las decisiones metodológicas que el profesorado ha de tomar durante las clases de lengua extranjera a adultos.

\section{Objetivos}

El objetivo principal de este estudio es indagar en las emociones tanto positivas como negativas que el alumnado adulto experimenta en la clase de LE para fundamentar las decisiones metodológicas del profesorado. Más concretamente, se pretende profundizar en el contexto del Nivel Intermedio B2.2. de la Escuela Oficial de Idiomas de Lucena con el fin de comprender los desencadenantes de estas emociones, las circunstancias en las que se producen y las implicaciones para las clases. Por otra parte, la formación del Grupo de Trabajo incluía objetivos para el profesorado, para el aula y para el centro.

Cuando se formó el Grupo de Trabajo en la Escuela Oficial de Idiomas de Lucena, los objetivos que el profesorado deseaba alcanzar consistían en la ampliación de su conocimiento en torno al disfrute y la ansiedad; la familiarización con las metodologías mixtas de investigación a través del desarrollo de métodos cuantitativos y cualitativos que permitirán elaborar un informe detallado del nivel de disfrute y ansiedad padecido por el estudiantado de la EOI de Lucena; el conocimiento de los niveles de ansiedad y disfrute que padece el alumnado adulto y los factores que los provocan; el conocimiento de las percepciones del alumnado; el conocimiento de las herramientas necesarias para diseñar y poner en marcha las actividades que fomentan el disfrute del alumnado adulto; y el conocimiento de las herramientas necesarias para diseñar y poner en marcha las actividades que disminuyen los niveles de ansiedad en el alumnado adulto.

Las repercusiones que estos objetivos tienen en el aula son el conocimiento detallado de las causas y detonantes de la ansiedad y el disfrute en el centro, el fomento de las actividades que promueven el disfrute, la concienciación sobre las actividades que causan ansiedad, y la aplicación de un enfoque metodológico y de estrategias que consiguen la reducción de las emociones negativas.

El objetivo del centro con este Grupo de Trabajo es que los profesores participantes puedan analizar todos los fenómenos de los aprendientes y sus realidades, comprender y familiarizarse con las experiencias que causan disfrute y/o ansiedad. De esta manera, se pueden fundamentar las decisiones metodológicas tomadas en las clases de Inglés como LE en la Escuela de Idiomas de Lucena.

\section{Desarrollo de la innovación}

Para desarrollar esta metodología innovadora fundamentada en el estudio exhaustivo de las necesidades emocionales del alumnado adulto de la Escuela Oficial de Idiomas de Lucena, se elaboró en primer lugar un cuestionario, que se revisó y distribuyó entre el alumnado de los miembros del Grupo de Trabajo por 
medio de los Formularios de Google. Tras la cumplimentación del cuestionario, se procedió a su análisis a través del programa estadístico IBM SPSS y a la elaboración de informes ilustrados mediante gráficas con los resultados relativos al nivel de FLE y FLCA del alumnado adulto del centro. Con esta información, las profesoras diseñaron tareas acordes para fomentar el disfrute y disminuir la ansiedad en sus aprendientes.

El instrumento utilizado en este estudio fue adaptado de la validación de un cuestionario piloto anterior en la Escuela Oficial de Idioma Xauen, en Jaén. El cuestionario actualizado constaba de varias secciones. En primer lugar, indagaba en las características sociodemográficas. En segundo lugar, los participantes debían indicar en qué medida estaban de acuerdo con los 21 ítemes de FLE y 8 de FLCA, basados en el cuestionario de Dewaele y MacIntyre (2014). Las respuestas posibles correspondían a una escala Likert de 5 variables $(1=$ totalmente en desacuerdo; $2=$ en desacuerdo; $3=$ indeciso/a; $4=$ de acuerdo; $5=$ muy de acuerdo). En tercer lugar, el instrumento incluía 3 preguntas de corte cualitativa sobre las experiencias personales del alumnado con respecto al disfrute y la ansiedad. Por último, se incluían preguntas relativas al uso de la lengua fuera de clase y en clase y a su predisposición a la comunicación.

Antes de difundir el cuestionario a través del enlace de Formularios de Google en las plataformas de aprendizaje en línea del profesorado y dejar tiempo para cumplimentarlo en clase, el instrumento fue revisado por dos expertos en Didáctica de la Lengua Extranjera y los 5 miembros del Grupo de Trabajo. La mayoría de las modificaciones que se llevaron a cabo estuvieron relacionadas con la formulación de las preguntas. Por ejemplo, en lugar de "8.6. Está guay saber hablar una LE", se propuso "8.6. Me resulta atractiva la idea de hablar una LE" o en el caso de "8.22. Incluso si estoy bien preparado/a para la clase de LE, me siento estresado/a" se sugirió "8.22. Aun estando bien preparado/a para la clase de LE, me siento estresado/a".

Los datos obtenidos se exportaron al programa estadístico IBM SPSS, a las variables de cada ítem se les asignó un valor para que el programa pudiera así realizar las sumas de FLE y FLCA y resumir la totalidad de los datos.

Por último, se resumieron los datos en informes ilustrados mediante gráficas con los resultados relativos al nivel de FLE y FLCA del alumnado adulto del centro. Con esta información, las profesoras diseñaron tareas acordes para fomentar el disfrute y disminuir la ansiedad en sus aprendientes.

\section{Resultados}

Un total de 190 estudiantes de inglés, francés y alemán de edades comprendidas entre los 14 y los 70 años rellenaron el cuestionario. No obstante, en esta comunicación, se analizan concretamente los resultados de los 25 aprendientes de Inglés como LE de Nivel Intermedio B2.2. en cuanto a FLE y FLCA, pese a que el Grupo de Trabajo analizó una gama de datos más amplia como se ha mencionado anteriormente.

\section{Análisis del disfrute (FLE)}

El alumnado tiene una percepción positiva de la clase de lengua extranjera dado que todos opinan que sus compañero/as son agradables, que hay buen ambiente en la clase de lengua extranjera, que la clase de lengua extranjera es divertida y a todos les resulta atractiva la idea de hablar una lengua extranjera. Además, las percepciones que el estudiantado tiene del profesorado son destacables en especial puesto que la inmensa mayoría opina que el/la docente es simpático/a, le apoya y anima en su proceso de enseñanza-aprendizaje. En general, disfrutan la clase de lengua extranjera, se ríen y se divierten en clase. 
Con respecto al progreso que advierten, el $88 \%$ afirma que ha aprendido a expresarse mejor en la lengua extranjera y el $96 \%$ asevera que ha aprendido cosas interesantes en la clase de lengua extranjera. Destaca asimismo que todos son conscientes de que cometer errores es parte del proceso de aprendizaje. No obstante, hay aspectos que se pueden mejorar. Un porcentaje considerable está en desacuerdo o indeciso con las afirmaciones "Soy un miembro valorado de la clase de lengua extranjera" y "Hay complicidad entre nosotros". Asimismo, destaca la variedad de respuestas con respecto a la afirmación "Me siento como si fuera una persona distinta en la clase de lengua extranjera". A la luz de estos datos, se entiende que, pese a estos últimos ítems susceptibles de mejora en cuanto al disfrute del alumnado, el estudiantado se muestra contento y satisfecho con la enseñanza que se lleva a cabo en la Escuela Oficial de Idiomas de Lucena.

En cuanto a las ocasiones en las que disfrutaron especialmente la clase de lengua extranjera destacan sobre todo las que implican la participación en juegos o actividades lúdicas, el desarrollo de relaciones humanas y la realización de actividades de producción y coproducción oral (Fig.1). Finalmente, tras la suma de todas las variables del cuestionario, se concluye que el disfrute experimentado en la Escuela Oficial de Idiomas de Lucena es de 92,84 en un rango entre 21 y 105, presentándose así resultados muy elevados en la escala de disfrute (FLE).

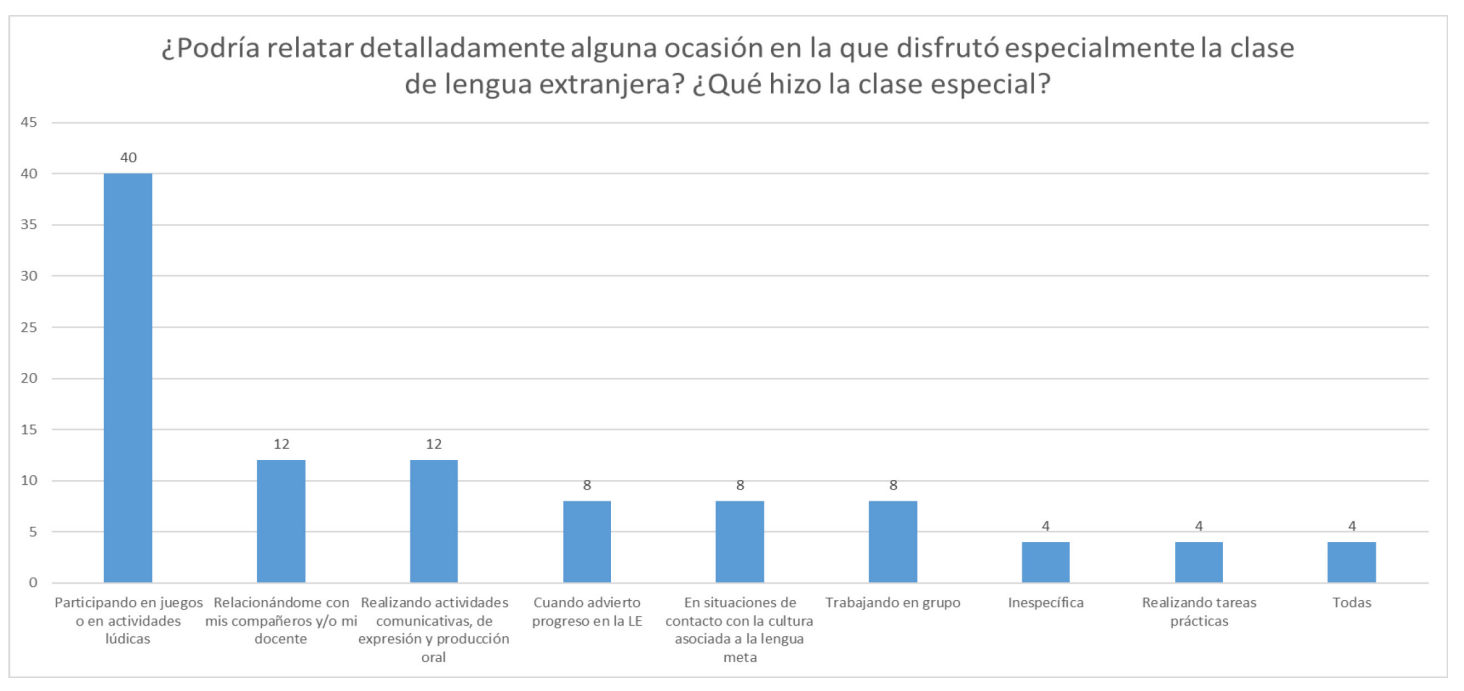

Fig. 1 Ocasiones en que el alumnado de B2.2. disfrutó especialmente la clase de inglés como LE

\section{Análisis de la ansiedad (FLCA)}

Como se puede observar en las gráficas inferiores, es inviable generalizar los datos obtenidos en cuanto a la experimentación de la ansiedad puesto que las respuestas con respecto a cada variable son muy diversas. 


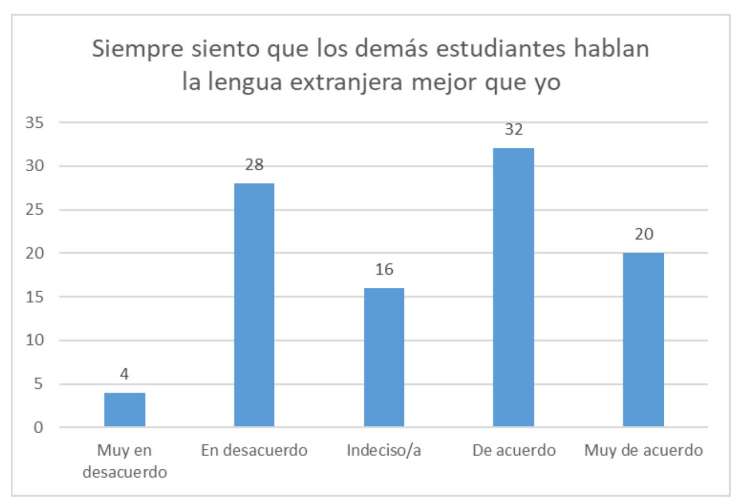

Fig. 2 Autopercepción del nivel en la LE con respecto a sus compañeros

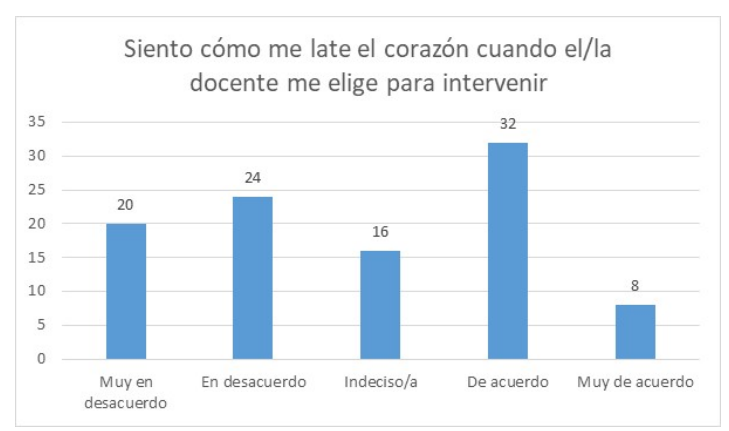

Fig. 3 FLCA cuando se les elige para intervenir en clase

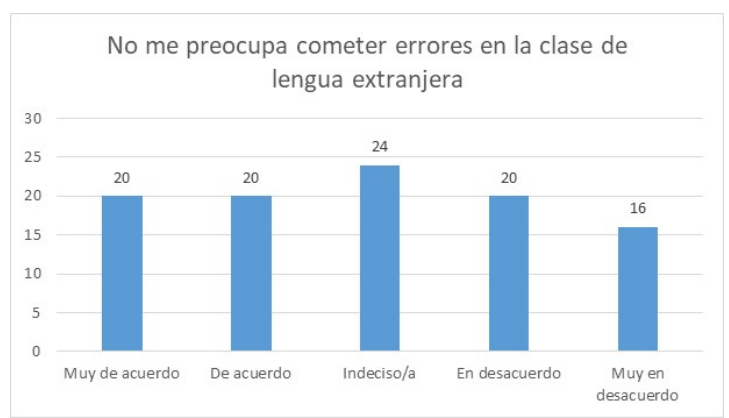

Fig. 4 FLCA al cometer errores en clase

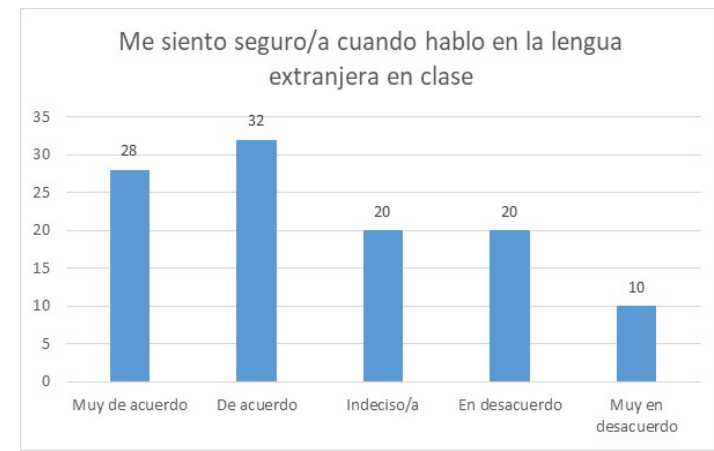

Fig. 5 Seguridad al hablar en clase 


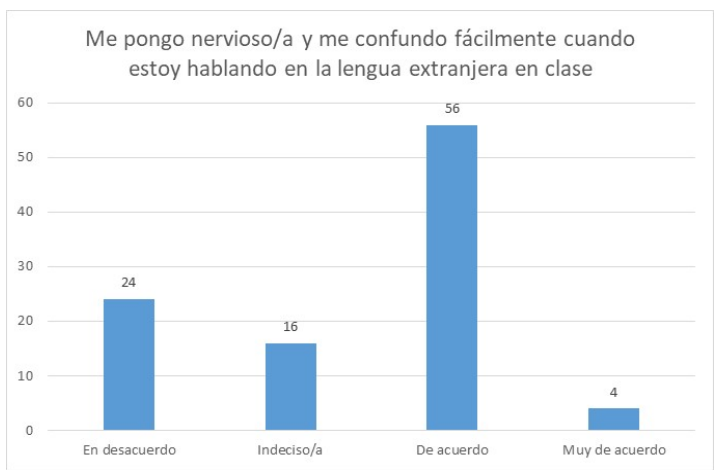

Fig. 6 FLCA al hablar la LE en clase

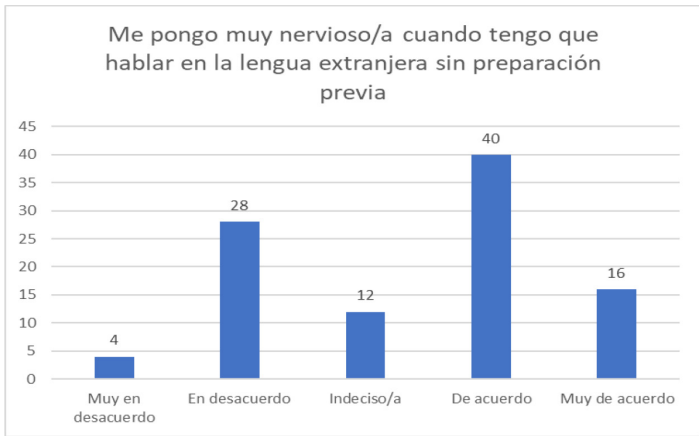

Fig. 7 FLCA sin prepararse previamente

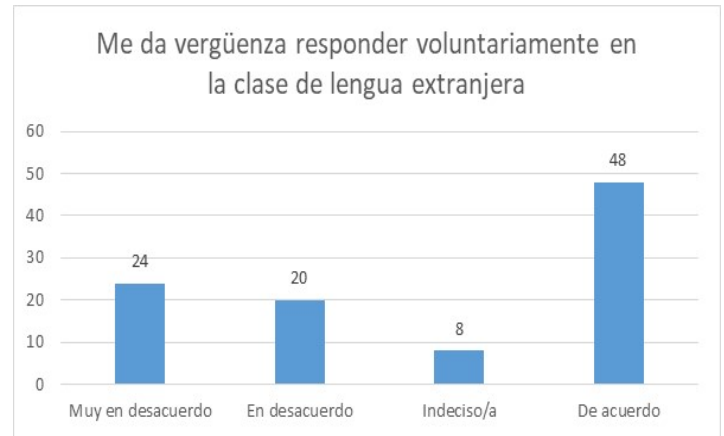

Fig. 8 Vergüenza a responder voluntariamente

Tras la suma de las variables y los resultados, la media de ansiedad experimentada por el alumnado es de 24,80 en un rango de 10 a 40 en la escala de ansiedad (FLCA). 


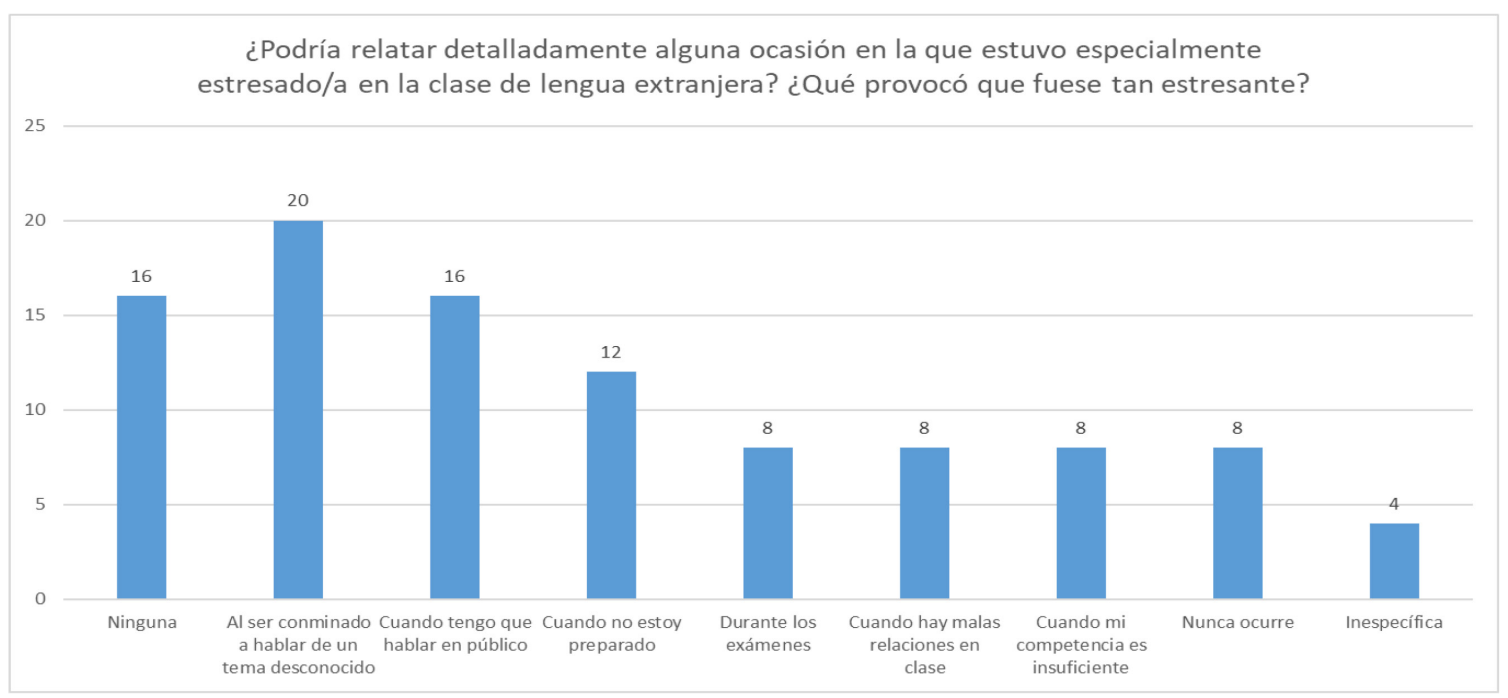

Fig. 9 Ocasiones en que el alumnado de B2.2. se estresó especialmente en la clase de inglés como LE

Cuando los participantes contestaron la pregunta cualitativa relativa a la ansiedad y estrés que padecen, gran parte sostuvo que no solía pasar. De los que respondieron con casos más concretos, señalaron que las situaciones que provocan más estrés y FLCA son el hecho de ser conminado a hablar de un tema desconocido, tener que hablar en público y la falta de preparación.

\section{Comparación entre disfrute (FLE) y ansiedad (FLCA)}

Cuando se le preguntó al alumnado por situaciones que disfrutara pese al esfuerzo, la mayoría se refirió a la realización de tareas de producción y coproducción oral. El disfrute experimentado en la Escuela Oficial de Idiomas de Lucena por parte de sus 25 participantes es de 92,84 en un rango entre 21 y 105 , presentándose así resultados muy elevados en la escala de disfrute (FLE) mientras que la media de ansiedad experimentada por el alumnado es de 24,80 en un rango de 10 a 40 en la escala de ansiedad (FLCA). Estos resultados indican que el estudiantado percibe las clases positivamente, aunque también experimenta un grado de ansiedad considerable. La presencia del disfrute no está directamente relacionada con la ansiedad y viceversa. Por lo tanto, los datos sugieren que el alumnado puede experimentar el disfrute y la ansiedad como dos variables independientes.

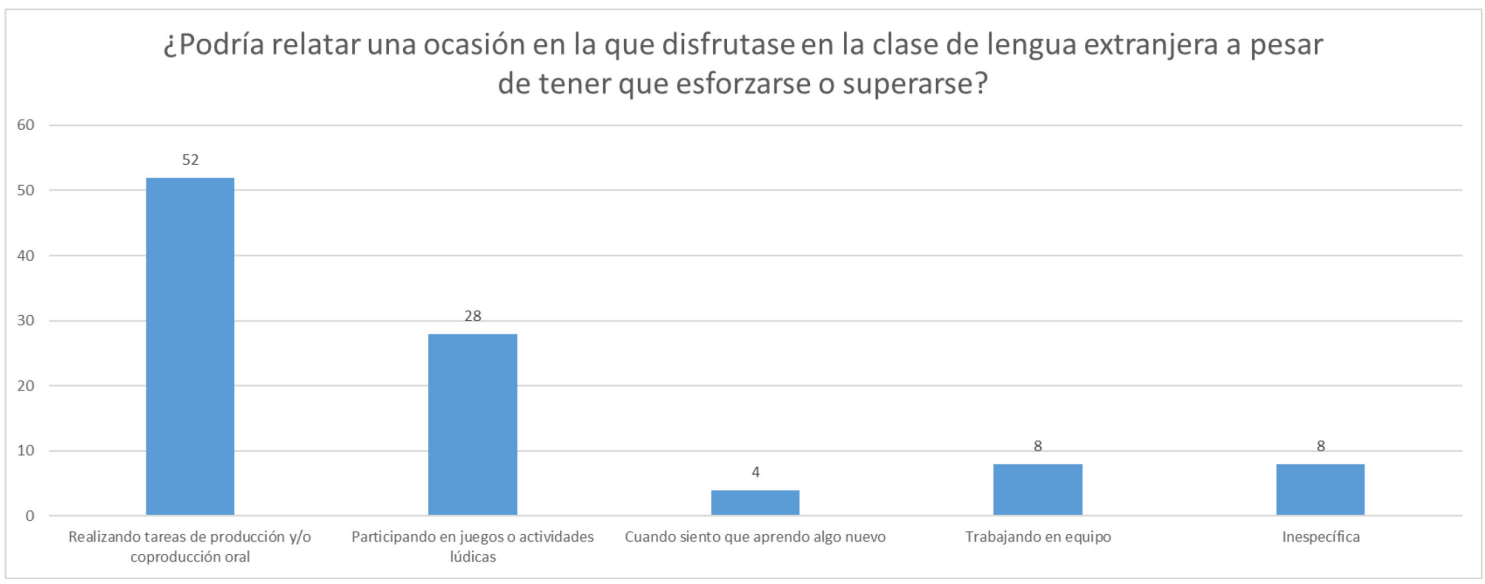

Fig. 10 Ocasiones en que el alumnado de B2.2. disfrutó pese al esfuerzo o superación 
Los resultados de la pregunta cualitativa sobre la experimentación de FLE y FLCA conjuntamente muestran el dinamismo mencionado en la introducción: es posible experimentar ambas emociones, aunque la presencia de una no implica la ocurrencia de la otra y viceversa. Además, estos resultados concuerdan con las investigaciones de Boudreau et al (2018), donde indican la diferencia entre el placer y el disfrute. El placer se centra en satisfacer las necesidades mientras que el disfrute se caracteriza por los retos y el progreso. El percibimiento de las emociones de esta manera establece una relación compleja entre FLE y FLCA, en la que un poco de FLCA es necesaria para producir experiencias agradables (Boudreau et al., 2018). Por estas razones, el papel que las emociones tienen en el proceso de aprendizaje de una LE es esencial y el profesorado debería analizar el FLE y la FLCA con el fin de incentivar experiencias desafiantes que conlleven FLE en la clase de LE.

\section{Decisiones metodológicas tras el análisis del disfrute y la ansiedad}

Las implicaciones de estos resultados son que la metodología utilizada en clase ha de integrar actividades lúdicas, relaciones humanas y oralidad (Fig.1) a la par que evita, en cierta medida, obligar al alumnado a hablar en público de temas desconocidos cuando no están preparados. Estas han de evitarse en cierta medida solamente puesto que las tareas de producción y coproducción oral producen gran satisfacción personal al alumnado pese al esfuerzo que suponen. Los resultados parecen indicar que la metodología más apropiada es el trabajo cooperativo.

Como los resultados obtenidos con respecto a FLE indican que la participación en juegos o actividades lúdicas, el desarrollo de relaciones humanas y la realización de actividades de producción y coproducción oral (Fig.1) son las tareas que más fomentan las emociones positivas, las profesoras del Grupo de Trabajo diseñaron una metodología que integrase estas preferencias.

En el caso de los estudiantes de inglés de Nivel Intermedio B2.2., se ha fomentado a través de tareas cooperativas lúdicas. Un ejemplo concreto es la realización de exámenes cooperativos a través de escape boxes. Para ello, la clase se divide en tres equipos, a cada uno se le asigna una caja que ha de solucionar eligiendo el símbolo correspondiente a la respuesta correcta en preguntas tipo examen (Fig.11). Es decir, la respuesta correcta tiene un símbolo asignado que les lleva por el camino adecuado dentro de un laberinto (Fig. 12). 


\section{We haven't done many researches into the subject yet.}

in

We haven't done researches into the subject yet

(4)

We haven't done much research into the subject yet

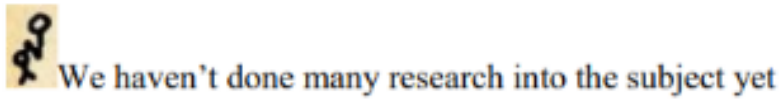

2. News are very disturbing.

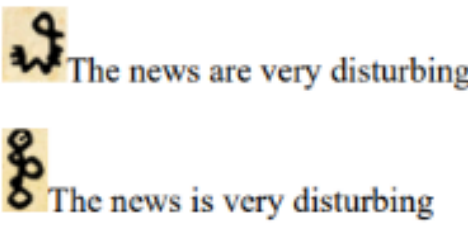

8 New is very disturbing

Fig. 11 Preguntas escape boxes

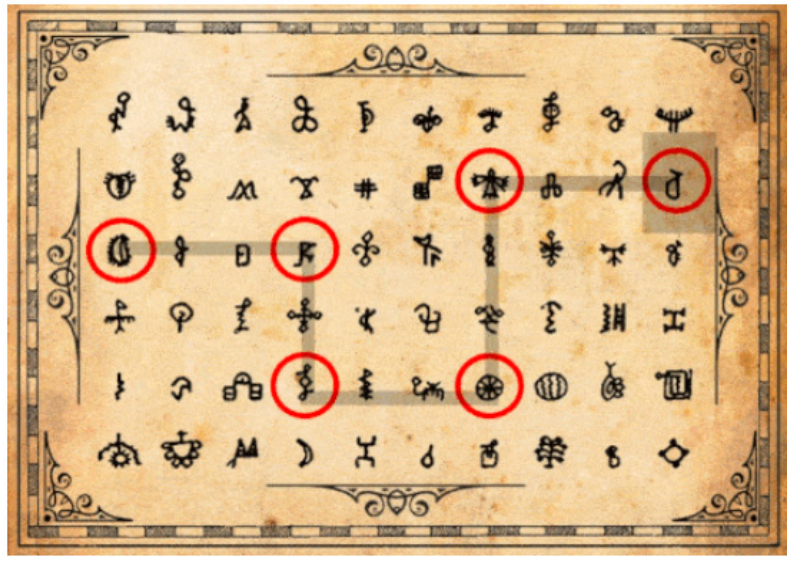

Fig. 12 Ejemplo recorrido imanes para extraer pista

Los estudiantes se encuentran con 3 cajas con símbolos extraños en su parte superior (Fig. 12), un rotulador y la hoja de pregutas tipo examen. Su objetivo es sacar un tornillo de la caja para conseguir acceder a la siguiente prueba. Para ello, la caja tiene un agujero del que tienen que extraer el tornillo con la pista sin intentar abrirla manualmente, usando solo el material que se les ofrece. Tendrán que encontrar los imanes que sobresalen en ella y seguir el camino arrastrándolos por un recorrido según las respuestas correctas.

El funcionamiento de la caja (Fig.13) se caracteriza por tener un tornillo dentro con la clave para la siguiente prueba, que obtienen uniendo los símbolos de las respuestas correctas en la hoja provista. Si aciertan, al mover la ficha compuesta de imanes de neodimio, mueven el tornillo que hay dentro de la caja hasta el final del laberinto. 


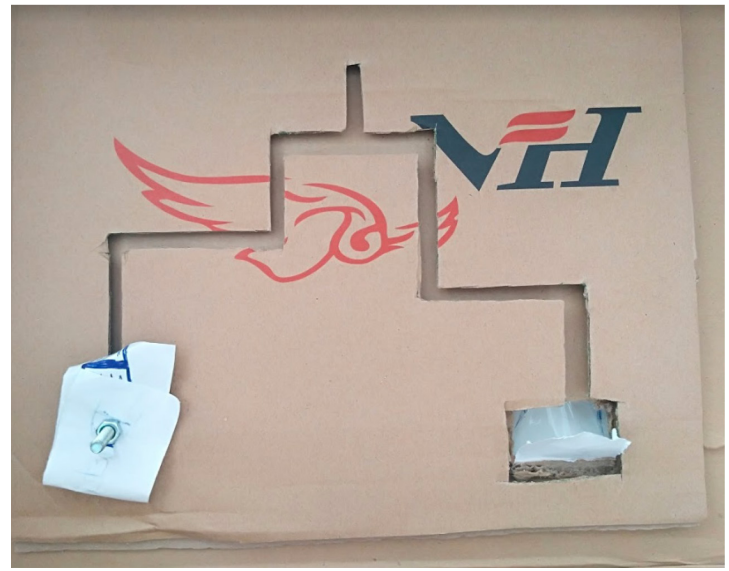

Fig. 13 Funcionamiento de la caja

De cada caja, se obtienen las siguientes pistas en esta tarea: New y el primer dígito para abrir la caja fuerte; English y el segundo dígito para abrir la caja fuerte ;Dictionary y el tercer dígito para abrir la caja fuerte.

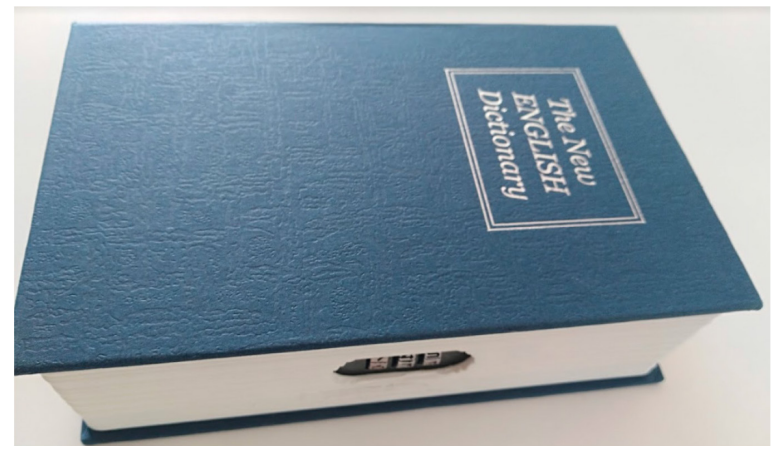

Fig. 14 Caja fuerte The New English Dictionary

La caja fuerte tiene la apariencia externa de un New English Dictionary (Fig.14). Cuando la encuentran e insertan los 3 dígitos, la abren y descubren 3 acertijos finales (Fig. 15), uno para cada equipo, sobre la cultura anglosajona.

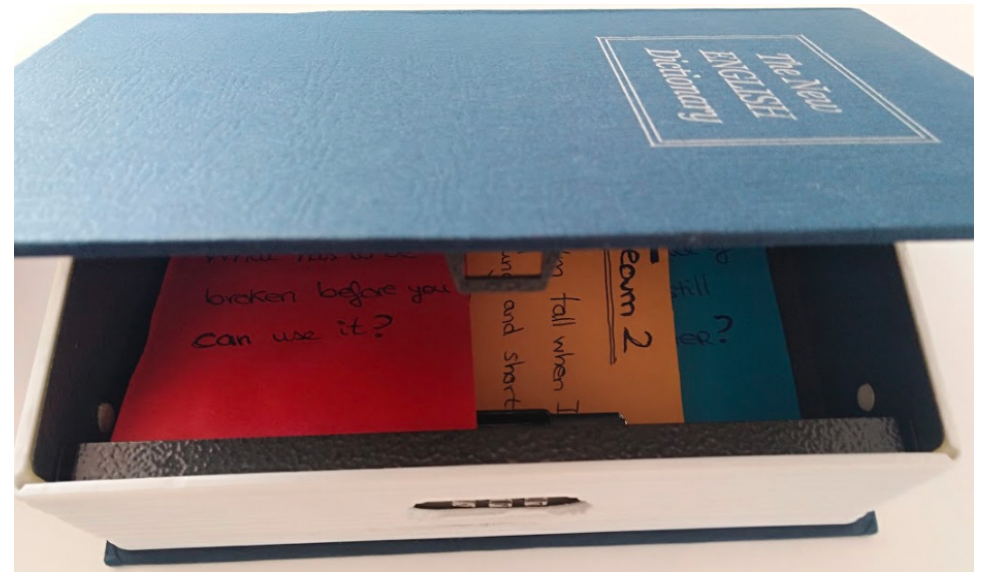

Fig. 15 Interior caja fuerte y acertijos finales

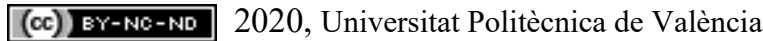

Congreso In-Red (2020) 
A nivel de escuela y no solo del grupo de Nivel Intermedio B2.2., en la plataforma Colabor@ de la Junta de Andalucía (2020) cada participante del Grupo de Trabajo describió las tareas que puso en marcha en clase basándose en los datos obtenidos (Fig.16). Se diseñaron tareas que estimularon al alumnado muy positivamente tales como un Concurso de Talentos, un Kahoot con las bromas propias de clase, un concurso de memes y una guerra de barquillos entre otras.

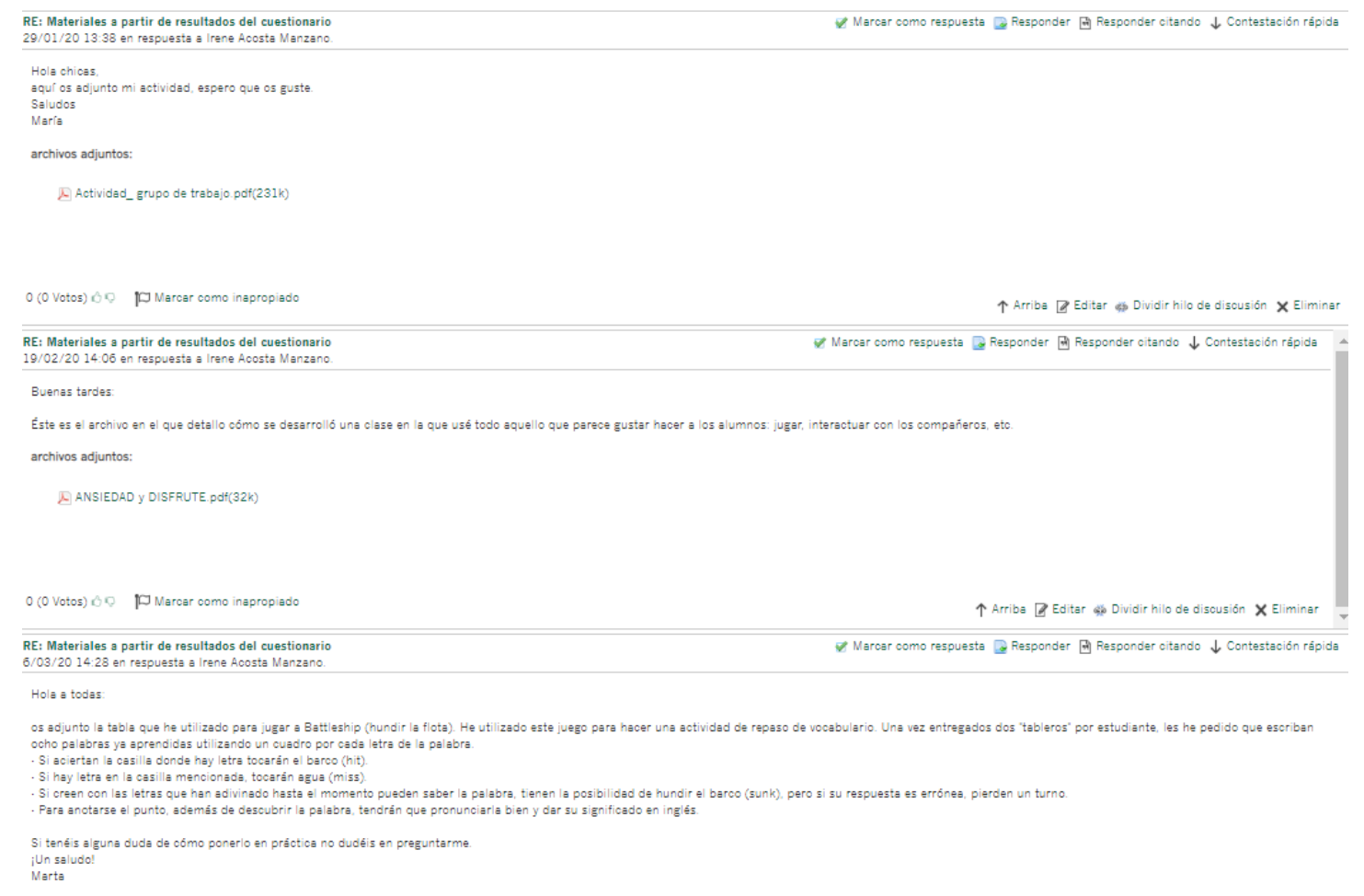

Fig. 16 Intervenciones en Colabor@

\section{Conclusiones}

Gracias a las indagaciones en el campo de las emociones positivas y negativas en el aprendizaje de LE por el Grupo de Trabajo de la Escuela Oficial de Idiomas de Lucena, se han podido fundamentar metodológicamente las decisiones del profesorado y se ha contribuido a la comprensión de las percepciones del disfrute y la ansiedad del alumnado. Los resultados de los 25 aprendientes de Nivel Intermedio B2.2. de inglés y de los 190 participantes de la escuela han permitido la identificación de las fuentes que ocasionan FLE y FLCA y la adaptación metodológica del proceso de enseñanza-aprendizaje según las emociones que el alumnado experimenta en clase.

Este estudio no solo ha permitido identificar las fuentes de disfrute y ansiedad en la clase de LE sino que también ha profundizado en las circunstancias en las que se producen, y sus implicaciones. El profesorado participante, por su parte, se ha visto beneficiado ya que ha ampliado sus conocimientos en torno a su alumnado, FLE y FLCA, se ha familiarizado con metodologías mixtas de investigación y con herramientas para el diseño de tareas que fomentan FLE y disminuyen los niveles de FLCA en alumnado adulto. Las repercusiones del aprendizaje del profesorado en el aula se reflejan en su preparación para tomar decisiones argumentadas en las clases de LE en la Escuela de Idiomas de Lucena. 
Las implicaciones de los resultados obtenidos concretamente en el grupo de Nivel Intermedio B2.2. de inglés son la necesidad de integrar actividades lúdicas, relaciones humanas y oralidad (Fig.1) dado que son los que fomentan el disfrute en mayor medida. Asimismo, con respecto a FLCA, el alumnado indica que ser conminados a hablar en público de temas desconocidos, sobre todo cuando no están preparados, les produce ansiedad (Fig.9). No obstante, las tareas que incluyan la oralidad en público han de evitarse únicamente en cierto modo puesto que las tareas de producción y coproducción oral producen gran satisfacción personal al alumnado pese al esfuerzo que suponen (Fig.10). En conclusión, el análisis de FLE y FLCA contribuye considerablemente a la obtención de conocimientos para el diseño de materiales diseñados según las necesidades de un alumnado tan poco estudiado tanto en la enseñanza de LE como general como es el adulto no universitario.

\section{Referencias}

BOUdREAU, C. H. E., MACINTYRE, P. D. y DEWAELE, J.-M. (2018). "Enjoyment and Anxiety in Second Language Communication: An Idiodynamic Approach" en Second Language Learning and Teaching, 8, 1, p. 21-45.

DEWAELE, J.-M. y MACINTYRE, P. D. (2014). "The two faces of Janus? Anxiety and enjoyment in the foreign language classroom" en Studies in Second Language Learning and Teaching, 2, 2, p. 237-274.

DEWAELE, J.-M. y MACINTYRE, P.D. (2016). "Foreign language enjoyment and foreign language classroom anxiety: The right and left feet of FL learning?" en MacIntyre, P.D., Gregersen, T. y Mercer, S. Positive psychology in SLA. Bristol: Multilingual Matters. p. 215-236.

DEWAELE, J.-M. y MACINTYRE, P. D. (2019). "The Predictive Power of Multicultural Personality Traits, Learner and Teacher Variables on Foreign Language Enjoyment and Anxiety" en Evidence-Based Second Language Pedagogy, Routledge, p-263-286.

DEWAEle, J.-M., MACINTYRE, P., BOUdREAU, C., y DEWAELE, L. (2016). "Do Girls Have All the Fun? Anxiety and Enjoyment in the Foreign Language Classroom" en Theory and Practice of Second Language Acquisition, 2, 1, p- 41-63.

DEWAELE, J.-M., y ALFAWZAN, M. (2018). "Does the Effect of Enjoyment Outweigh That of Anxiety in Foreign Language Performance?" en Second Language Learning and Teaching, 8, 1, p. 16-21.

DEWAELE, J.-M., y DEWAELE, L. (2017). "The Dynamic Interactions in Foreign Language Classroom Anxiety and Foreign Language Enjoyment of Pupils Aged 12 to 18. A Pseudo-Longitudinal Investigation" en Journal of the European Second Language Association, 1, 1, p. 12-22.

FREDRICKSON, B. L. (2003). "The value of positive emotions" en American Scientist, 91, 4, p. 330-335.

GREGERSEN, T., MACINTYRE, P. D., y MEZA, M. D. (2014). "The motion of emotion: Idiodynamic case studies of learners' foreign language anxiety” en Modern Language Journal, 98, p- 574-588.

JUNTA DE ANDALUCIA. Colabor@ 3.0. < https://colaboraeducacion30.juntadeandalucia.es/educacion/colabora/> [Consulta: 09 de marzo de 2020]

LI, C. (2019). "A Positive Psychology perspective on Chinese EFL students' trait emotional intelligence, foreign language enjoyment and EFL learning achievement" en Journal of Multilingual and Multicultural Development. 0,0, p. 1-18.

MACINTYRE, P. D. (1999). "Language anxiety: A review of the research for language teachers. en Young, D. J. (Ed.), Affect in foreign language and second language teaching: A practical guide to creating a low-anxiety classroom atmosphere (pp. 24-45). Boston: McGraw-Hill.

MACINTYRE, P. D., y GREGERSEN, T. (2012). "Emotions that facilitate language learning: The positivebroadening power of the imagination" en Studies in Second Language Learning and Teaching, 2, 2, p. 193-213.

(cc) EY-NC-ND 2020, Universitat Politècnica de València

Congreso In-Red (2020) 
MACINTYRE, P. D., y GREGERSEN, T. (2014). "Editorial" en Studies in Second Language Learning and Teaching. 4, 2, p. 149-152.

PAVELESCU, L. M., y PETRIĆ, B. (2018). "Love and enjoyment in context: Four case studies of adolescent EFL learners" en Studies in Second Language Learning and Teaching. 8, 1, p. 73-101.

SAITO, K., DEWAELE, J. M., ABE, M. y IN'NAMI, Y. (2018). "Motivation, Emotion, Learning Experience and Second Language Comprehensibility Development in Classroom Settings: a Cross-Sectional and Longitudinal Study" en Language Learning. 68, 3, p. 709-743. 